\title{
Research and Analysis of Mathematical Model for photo voltaic Power Generation Network
}

\author{
Longsong Duan ${ }^{1,{ }^{*}}$, Delong Wan ${ }^{2, b}$ \\ ${ }^{1}$ Nanchang University of Gongqing College, Jiujiang City, China; \\ ${ }^{2}$ Science and Technology College of Nanchang Hangkong University, Jiujiang City, China. \\ *Corresponding author Email:adls78@sina.com , b249245952@qq.com
}

Keywords:photo voltaic power generation, Reactive power compensation, Power, panel

\begin{abstract}
With the development of solar power generation, the research of high-power photo voltaic grid-connected systems has become a hot topic. The establishment of mathematical models for photo voltaic arrays and photo voltaic grid-connected converters has important research on photo voltaic grid-connected technologies. This paper studies the low-voltage ride-through control strategy of photo voltaic grid-connected system under symmetrical drop, and realizes fast and smooth switching with the normal operation mode of the grid. It has important practical significance and theoretical research basis for future work and research.
\end{abstract}

\section{Introduction}

PV convector is the core equipment of photo voltaic power generation grid-connected system. Its main function is to convert the DC electricity generated by the PV pool plate into AC energy that can be integrated into the grid, and at the same time, the performance meets the harmonic content and power factor of the grid-connected current. Standard requirements. However, solar energy resources are characterized by random intervals, and output fluctuations are large and irregular. With the increase of the access ratio of photo voltaic systems[1], the impact of large-scale photo voltaic grid-connected systems on the power system is also increasing. Therefore, it is necessary to establish a photo voltaic grid-connected system model to analyze its operating characteristics[2].

\section{Characteristics of photo voltaic power generation}

Photo voltaic power grid-connected system control adopts the constant voltage method, and is set according to the maximum power point parameter of the photo voltaic cell board. The control in the grid-connected mode adopts a double-closed-loop control strategy based on the voltage-oriented feedback loop of the power grid and the feedback loop of the grid-connected current feedback. The voltage outer loop controls the intermediate DC voltage to track the given MPPT voltage; the inner loop control AC output current. The outer ring PI regulation output is the d-axis current command id of the current loop, that is, the size of the active current, the q-axis current inner loop controls the network-side power factor, and the q-axis current is set to zero, that is, the converter is operated at the unit power. Factor status[3].

With the rapid development of solar photo voltaic power generation, the grid connection of high-power photo voltaic power generation has become a research hot spot. The key issues in the photo voltaic grid-connected system exist in two aspects. One is the research on the topological structure of the high-power photo voltaic generation grid-connected system. Selecting the appropriate topology is the basis of the device hardware, and the key point is that it can run safely and stably and reduce the number of systems[4]. The energy loss maximizes the photo voltaic power generation efficiency. The second is the distributed MPPT technology, which is the maximum power tracking technology for large-scale solar panels. MPPT control technology is mainly divided into centralized control and decentralized control. At present, the centralized control method is relatively mature, and 
it is also the most widely controlled technology, but for high-power photo voltaic power plants, centralized control can not meet the control needs of the system, and the efficiency is low. Distributed control technology is the focus of future research[5].

\section{Mathematical model of grid-connected PV power generation}

With the rapid development of distributed generation and the continuous improvement of user-side demand, it is imperative to properly select DG in combination with the user-side load characteristics, the specific geographic location, environmental conditions, and resource distribution. By analyzing the output mathematical models of different DG and the interaction between DG and energy storage elements, they are investigated to a series of effects on the power quality and voltage stability of the distribution network. The research on distribution network is based on the calculation of power flow. The access of different DG and energy storage elements will have different effects on the distribution of distribution network power flow. Therefore, in this paper, the power factor is used as the influence factor to analyze the mathematical models of different DG under different power generation conditions, and the analysis of different types of the same DG combined demand will have different effects on the current distribution of the system. Users can choose DG according to their actual situation and research direction[6].

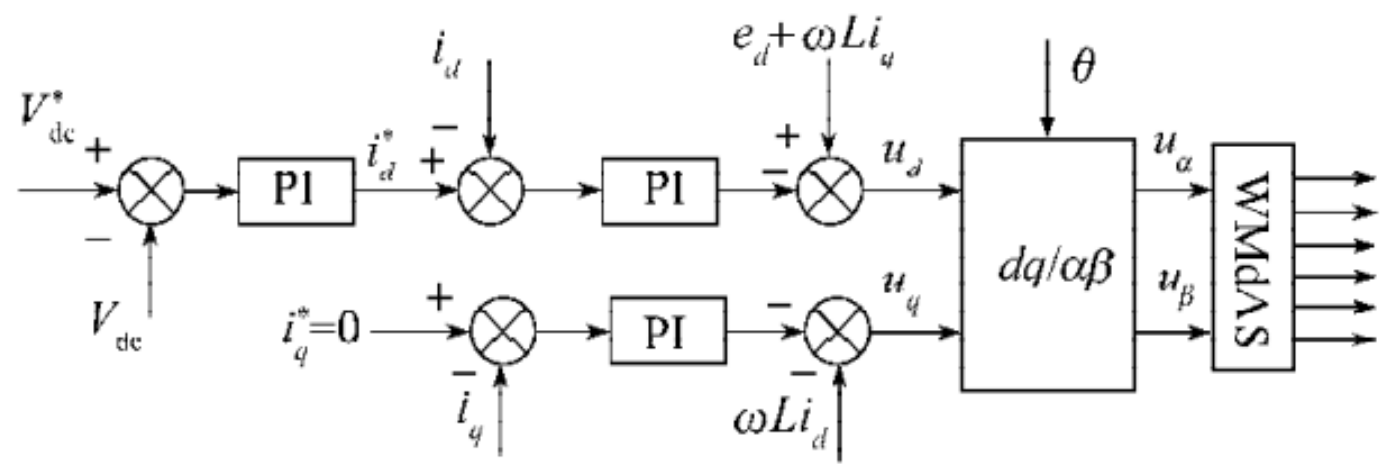

Fig. 1 Photo voltaic controller logic circuit diagram

\subsection{PV Converter Low Voltage Ride Through Control}

Photo voltaic power system low voltage ride through (LVRT) means that when the voltage at the PV power station access point falls, the PV power plant can maintain the grid-connected operation status and provide a certain amount of reactive power to the grid to support grid voltage recovery until the access point. The voltage returns to normal and thus "passes through" the period during which the voltage falls[7].

Both domestic and foreign photo voltaic grid-connected rules require that PV power plants provide reactive power in the event of a power grid failure (such as a voltage drop) to regulate the system voltage. Under the condition of not adding hardware devices, low voltage ride-through of PV stations can be achieved by changing the control strategy of PV inverses. The key to low voltage ride-through of PV inverse is that the PV inverse grid-connected current is limited to the crosscurrent protection threshold during grid faults[8]. During grid voltage dips, PV grid-connected converters can operate in STATCOM mode. Calculate the reactive power that needs to be output to the grid based on the degree of grid voltage dips. The larger the voltage drop of the power grid, the greater the reactive power that needs to be added to the grid, and thus the more reactive support provided to the grid, which is beneficial to the restoration of the grid voltage;such as:;

$$
\cos \theta=\sin \alpha \cos \beta+\cos \alpha \sin \beta \cos (\gamma-A)
$$

\subsection{Research on photo voltaic Power Factor}

It improves on the traditional double closed-loop control strategy and uses a network pressure feed-forward control link to calculate new reference values for active and reactive currents, in which 
the active current reference value is directly given, and the reactive current reference value is When the result is taken from the voltage outer loop, the controller will continuously detect if the access point voltage has dropped. If there is no drop, the traditional double closed-loop control method is still maintained. The converter operates in the state of power factor of 1 . At this time, only the active power is delivered to the grid[9]; if the voltage at the grid connection point falls, the original double closed-loop control is disconnected. The outer voltage loop of the voltage converter is changed to the current single closed loop operation mode. Although there is no DC voltage outer loop control, the DC voltage can still be maintained at a stable value due to the output characteristics of the photo voltaic cell, but it will deviate from the previous one. The maximum power point voltage. When the control section of the grid voltage feed forward is put into operation, the ratio between the reactive current and the voltage drop depth should be reasonably controlled according to the photo voltaic grid-connected criterion, that is, the reference value of the reactive current is calculated according to the depth of the grid voltage drop. In this case, the converter operates in the reactive power compensation mode, and the reactive current is the main control object. By directly specifying the active current reference value of the converter for the rated current of the converter, the active and reactive current commands can be restarted. distribution.

\subsection{Double closed-loop control of photo voltaic grid-connected power generation}

PV grid-connected system converter control strategy, this article uses a double closed-loop control mode, the inner loop current control, the outer loop voltage control mode, the specific control block diagram shown in Figure 2. The current inner loop control strategy compares the output current of the converter with the set current, the voltage outer loop control compares the DC bus voltage with the given voltage, compares the feedback into the system, the d-axis component control is the DC voltage of the system, and the $\mathrm{q}$ axis The power factor of the component control system is PI-adjusted according to the feedback result of the system, and the control method of the SVPWM is used to control the on-off of the switching device of the converter to realize the control method for the converter. The power switch is a filter tube and the filter inductance is The switching ripple filter is the sum of the parasitic series resistance of the filter inductor, the turn-on voltage drop of the switch, and the line resistance. The pseudo DC bus voltage is the output voltage of the inverse bridge and the grid voltage is the grid current.

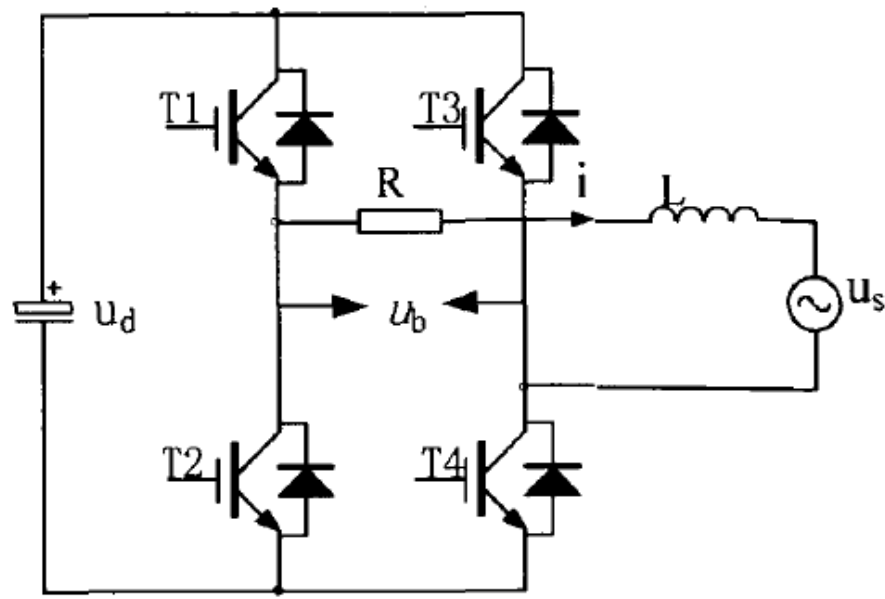

Fig. 2 Photo voltaic double closed-loop control circuit

\section{Research and improvement of the mathematical model of grid-connected photo voltaic generation}

The maximum power point tracking control of photo voltaic power generation In the photo voltaic power generation grid-connected system, the first consideration of the control strategy is the maximum power tracking problem of the solar panels, that is, the control of the solar panels is always in the state of maximum power generation. In the traditional maximum power tracking strategy, the hill climbing method is a classic algorithm. The meaning of the algorithm is that when the solar pool 
panel works normally, the voltage is used as the disturbance, and the output power is used as the observation and the system continuously performs the disturbance to seek the maximum value of the output of the system. . The specific control process is the disturbance of the voltage increase of the system at a certain moment, and the change of the output power of the observation system. If the output power of the system becomes larger, it means that the system is not at the optimal operating point, and the direction of the optimization and disturbance of the system is correct. The system voltage increase disturbance continues until the system output power no longer increases, and the system is at the optimal operating point. Similarly, when the loaded voltage increases, the system power decreases, indicating that the system needs a buck-boost disturbance. The optimization process is the same. Rational and booster disturbances. The advantage of the traditional hill climbing method is that the control strategy is simple and easy to implement, but the continuous optimization process will result in unavoidable power loss and reduce the efficiency of the system. At the same time, the initial search time in the tracking process is longer. It is difficult to determine the amount of voltage disturbance. In response to the above problems, the following improvements have been made to the traditional hill climbing method.

\subsection{Inverse Control Section}

\section{(1) Model control method classification}

The model of the inverse control link is usually given in the form of a control block diagram. The input quantities are the grid-side and array-side power, and the output is the modulation ratio and direction-of-arrival of the PWM control link or the output power of the inverse. Since the purpose of modeling the inverse control link is usually to verify and verify various control methods, the block diagrams of the existing control models are different. In addition to the usual proportional, integral and derivative control methods, some new ones can also be adopted. Control Method. Therefore, the models of the inverses are different, but depending on the purpose of the model control, there are three types of constant power factor control methods, constant voltage control methods, and active and reactive power decoupling control methods.

\section{(2) Constant power factor control method}

When the output current of the inverse and the grid voltage are not synchronized, that is, there is a phase difference, the inverse outputs reactive power. Due to the higher cost of inverse reactive power generation, the inverse's fixed output power factor must be controlled to be to ensure that it does not generate reactive power. The input of the inverse constant power factor control method can be the voltage or current on the grid side. By monitoring the phase difference between the current and voltage, the output power factor can be guaranteed to be constant.Repeated control and voltage feed forward can also be used. Other methods improve the accuracy of control .

\section{(3) Constant voltage control method}

For a photo voltaic power system that is connected to an AC system, if it needs to provide certain reactive power support for the power system, a constant voltage control method must be used. There is no technical difficulty in achieving a constant voltage control method on the AC side, and only the control target of the inverse can be modified. However, as mentioned above, it is not economical for the inverse to generate reactive power to keep the grid-connected voltage constant. Therefore, reactive power compensation equipment is generally used for voltage control. However, if a photo voltaic power generation system that supplies power directly to a DC load through a DC bus, in order to reliably supply power to the load, it is necessary to control the DC bus voltage constant.

\section{(4) Output power decoupling control method}

The output power control of the inverse is achieved by controlling its PWM signal. Directly adjusting the modulation ratio or phase-shifting angle of the PWM control signal of the inverse will affect the active and reactive power of the inverse output. Therefore, it is necessary to search for the solution of the active and reactive power output of the photo voltaic power generation system. Coupled control methods. However, the reactive power component iq of the current in the inverse and the active power component id are coupled to each other and cannot be controlled separately. The inverse output current should be decoupled first to separately control the active and reactive power of the output. 


\subsection{Power flow calculation model of photo voltaic power generation system}

Photo voltaic power sources mostly adopt constant power factor control. If there is no need to calculate the internal parameters of the photo voltaic power generation system, the photo voltaic power source can be equivalent to a PQ node in the power flow calculation.

If it is necessary to calculate the internal parameter values of the photo voltaic power generation system while calculating the power flow, a power flow calculation model for photo voltaic power generation systems including photo voltaic arrays, inverse bridges, and alternating current circuits including transformers and filters can be established. Literately solve the equations and the power flow of the grid, and finally obtain the power system power flow of the photo voltaic power generation system and the internal parameters of the photo voltaic power generation system.

photo voltaic power output has a random nature, and it can use stochastic power flow method to calculate the impact of lighting changes on system power flow, which can be used to express light intensity and photo voltaic power generation system output changes using beta probability function to obtain the following model $\alpha$ and $\beta$ as Beta distribution shape parameters; PM is the total output power of the PV array; RM is the maximum power that the PV array can output.

\subsection{Transient Stability Calculation Model of photo voltaic Power Generation System}

\section{(1) System model based on controlled source method}

The photo voltaic power generation system transient model is based on the above-mentioned photo voltaic cell and inverse model, and the key to its modeling lies in the use of suitable methods to connect photo voltaic cell, inverse, transformer, filter, and voltage stabilization capacitor and other component models. The entire system model. Specific methods include controlled source methods, current and voltage methods, and equation systems. This method divides the system into three parts: the photo voltaic array, the inverse, and the AC grid connection. The three are connected by an inverse and a transformer. Each part first establishes its own model, and regards the connection between the inverse and the transformer as the relationship between the controlled quantity and the controlled source, thereby linking each part through the relationship of the controlled source and obtaining the circuit model of the entire system. According to this model, the state equation of the system can be obtained, and then the dynamic equation of the system can be obtained by assuming that all the communication quantities are fundamental sine quantities.

\section{(2) Model based on current and voltage method}

Establishing a system model based on the relationship between the voltage and current quantities between the various parts of a photo voltaic power generation system is a relatively straightforward method. The model of the photo voltaic cell is based on its physical properties. The MPPT controller collects the voltage and current on the photo voltaic cell side. The grid-connected and reactive power controllers collect voltage and current on the grid side. Both control the SPWM generator at the same time. The inverse adopts a combination of feed forward and feedback control methods, and adds the model of the anti-jamming and boost filtering components of the previous stage. The circuit model of the entire photo voltaic power generation system can be obtained by directly connecting the above-mentioned parts of the model with a circuit connection or a voltage and current signal connection.

\section{(3) Models based on equations}

The equations of state of the various components of the photo voltaic power generation system are first established by using the equation set method, and the control block diagrams of the inverse and the MPPT control link are converted into the state equations, and the equations of state equations are obtained through simultaneous determination, that is, the entire photo voltaic power generation system mode.

The above model has both circuit and state equations, but the MPPT control and inverse control link need to be literately calculated under certain control strategies. The control process is complicated and difficult to express by circuit or state equation. The use of a program segment to represent this control process and embedding in the entire system model is a concise method that also applies to most simulation software. 


\section{Modeling of Island Protection in photo voltaic Power Generation System}

The island protection of photo voltaic power generation system is divided into active protection and passive protection. Active protection detects islands by periodically injecting disturbances into the grid and observing grid responses to disturbances. Periodic disturbances of active protection can have an adverse effect on the grid, and the real need to actively protect detected island states is often difficult to achieve. Passive protection detects the existence of islands by detecting voltage, frequency, zero-crossing, phase, and other system variables. However, when the photo voltaic system generates power and loads, the island state may not be detected. No matter which type of island protection is adopted, it can only be modeled after researching whether island protection can operate correctly under the state of is landing and other impacts of the grid, and embedded in the system model for simulation. Due to the existence of logical judgment, island protection usually appears in the entire system model as a program.

\section{Conclusion}

In this paper, in-depth study of traditional photo voltaic grid-connected mathematics structure was conducted, and a high-power photo voltaic grid-connected system was designed. In terms of grid-connected hardware, the three-phase voltage type full-bridge topology with single-stage energy conversion structure is used as the core structure of the grid-connected converter. In terms of software control strategy, the mathematical model of photo voltaic power generation is first established, using double The closed-loop control method controls the photo voltaic grid-connected system by SVPWM. At the same time, according to the maximum power tracking problem of photo voltaic power generation, this paper optimizes the design based on the traditional strategy of climbing-based optimization, and improves the speed and accuracy of optimization. The system performance is better, the output efficiency is higher, and it meets the requirements of the power grid.

\section{References}

[1]. Xiao Xinxin, Liu Dong, et al. An Overview of Distributed Power Supply System Access Grid Model. East China Electric Power. Vol. 43 (2008) No. 3, p. 76-81.

[2]. Guo Yong, Sun Chao, Chen Xin, et al. Research on Maximum Power Point Tracking in photo voltaic System. Power Electronics Technology. Vol. 26 (2009) No. 9, p. 21-23

[3]. Sun Chao, Guo Yong, Chen Xin. Research on Solar Charger in Independent photo voltaic System. Power Electronics Technology. Vol. 45 (2009) No. 10, p. 44-46.

[4]. Chen Ruliang, Cui Yan, Li Dayong, et al. photo voltaic module simulation model under non-uniform lighting conditions. Journal of System Simulation. Vol. 17 (2008) No.7 p. 1681-1690.

[5]. Liu Bangyin, Duan Shanxu, Kang Yong, et al. Modeling and analysis of photo voltaic module characteristics under local shadow conditions. Chinese Journal of Solar Energy. Vol. 32 (2008) No. 7, p. 188-192.

[6]. Yan Meiqin,Yu Shijie,Su Jianhui, et al. General Simulation Model of photo voltaic Array MATLAB with MPPT.Journal of System Simulation. Vol. 29 (2005) No.6, p. 1248-1251.

[7]. Wu Jie, et al. Research on photo voltaic grid-connected/independent power dual-purpose inverse power supply. Hefei: Hefei University of Technology. Vol. 34 (2005) No. 3, p. 215-218.

[8]. Wang Jinjin, et al. Research on photo voltaic grid-connected control method and system.University of Technology. Vol. 34 (2002) No.8, p. 116-119.

[9]. Zhao Wei, et al. Research on grid-connected photo voltaic power generation system. University of Technology. Vol. 25 (2003) No. 5, p.32-35. 